\title{
Probabilistic-based analysis for damaging features of fatigue strain loadings
}

\author{
M. F. Mod Yunoh, S. Abdullah \\ Centre for Automotive Research, Faculty of Eng. and Built Enviroments, Universiti Kebangsaan Malaysia \\ faridzyunus@gmail.com,shahrum@ukm.edu.my
}

\author{
M. H. M. Saad, Z. M. Nopiah, M. Z. Nuawi, S. S. K. Singh \\ Dept. of Mechanical and Materials, Faculty of Eng. and Built Enviroments, Universiti Kebangsaan Malaysia \\ hanifsaad@ukm.edu.my,zmn@uk.m.edu.my,mzn@ukm.edu.my,salvinder@ukm.edu.my
}

\begin{abstract}
This paper presents the behaviour of fatigue damage extraction in fatigue strain histories of automotive components using the probabilistic approach. This is a consideration for the evaluation of fatigue damage extraction in automotive components under service loading that is vital in a reliability analysis. For the purpose of research work, two strain signals data are collected from a car coil spring during a road test. The fatigue strain signals are then extracted using the wavelet transform in order to extract the high amplitude segments that contribute to the fatigue damage. At this stage, the low amplitude segments are removed because of their minimal contribution to the fatigue damage. The fatigue damage based on all extracted segments is calculated using some significant strain-life models. Subsequently, the statistics-based Weibull distribution is applied to evaluate the fatigue damage extraction. It has been found that about $70 \%$ of the probability of failure occurs in the $1.0 \times 10^{-5}$ to $1.0 \times 10^{-4}$ damage range for both signals, while $90 \%$ of the probability of failure occurs in the $1.0 \times 10^{-4}$ to $1.0 \times 10^{-3}$ damage range. Lastly, it is suggested that the fatigue damage can be determined by the Weibull distribution analysis
\end{abstract}

KEYwORDS. Fatigue damage; Features extraction; Probabilistic; Wavelet; Weibull distribution.

\section{OPEN ACCESS}

Citation: Yunoh, M. F. M., Abdullah, S., Saad, M. H. M., Nopiah, Z. M., Nuawi, M. Z., Singh, S. S. K., Determining probabilistic-based failure of damaging features for fatigue strain loadings, Frattura ed Integrità Strutturale, 46 (2018) 84-93.

Received: 18.01 .2018

Accepted: 20.05 .2018

Published: 01.10.2018

Copyright: (C) 2018 This is an open access article under the terms of the CC-BY 4.0, which permits unrestricted use, distribution, and reproduction in any medium, provided the original author and source are credited.

\section{INTRODUCTION}

$\mathrm{F}$ atigue life prediction under service loading remains a challenging problem in real life, especially in the automotive industry. In an automotive application, service loadings such as stress on a car wheel are in variable amplitude loading, as reported in Sonsino [1]. This situation leads to a need for developing a new approach to predict the reliability of 
the components that are subjected to the fatigue loading. The fatigue time histories often contain a major percentage of small amplitude cycles, and the fatigue damage for these cycles can also be small. Therefore, in many cases, the fatigue loading history is edited by removing these small amplitude cycles to produce representative and meaningful, yet economical testing, Stephens et al. [2].

Several fatigue data editing techniques have been developed for use in the time domain analysis, Abdullah et al. [3]. Some previous algorithms have been developed for eliminating low amplitude cycles in order to only retain the high amplitude cycles, El Ratal et al. [4]. In the frequency domain, the time history is a low pass filtered by the criterion that high-frequency cycles have small amplitude, which is are not damaging. The filtering method does not shorten the signal because it does not provide the time-based information, Nizwan et al. [5]. In addition, Abdullah et al. [3] have developed a method for data editing to shorten the strain signal in the time-frequency domain. In fatigue data editing, the behaviour of extraction segments also needs to be studied because it contributes many bits of information that can improve fatigue life prediction. To deal with uncertainties and variations in fatigue data, the statistical analysis, i.e. the probability analysis is the best approach that should be adopted. Zhao and Liu [6] use the approach of statistical aspects of the S-N curve by means of the Weibull distribution. This approach indicates that an appropriate distribution determination is the primary task for a rolling contact fatigue analysis. Tiryakioglu [7] uses the Weibull analysis in fatigue data and predicts the failure mechanisms due to cracks initiating from surface and interior defects.

The evaluation of fatigue damage extraction for automotive components under service loading is vital in the reliability analysis. Very few analysis methods have been developed to evaluate the fatigue damage extraction. A consistent description of the probability of damage occurrences is possible only if the damage distribution function is known. In this paper, two sets of strain signals from the real component in service are used in the fatigue feature extraction. The feature extraction, i.e. fatigue damage, is analysed using statistical inferences. The objective of this study is to determine the probabilistic-based failure of damage featured in the strain signals, and at the same time, validate them through the extraction process. By considering the significant statistical tools, features extraction is combined with the Weibull distribution analysis in order to obtain a better evaluation.

\section{THEORETICAL BACKGROUND}

\section{The Global Statistic}

he time series contains an explanation of a set of variables taken at equally spaced time intervals. A statistical analysis is normally used to determine the random signals and monitor the pattern of the analysed signals. The calculation of the root-mean-square (r.m.s.) and the kurtosis is very important in the fatigue signals in order to retain a certain number of the signal amplitude range characteristics. The r.m.s. value is the 2 nd statistical moment, is used to quantify the overall energy content of the signal. For discrete data sets the r.m.s. value is defined as:

$$
r . m . s=\left\{\frac{1}{n} \sum_{j=1}^{n} x_{j}^{2}\right\}^{1 / 2}
$$

While kurtosis is the signal 4th statistical moment, is a global signal statistic which is highly sensitive to the spikiness of the data. For discrete data sets the kurtosis value is defined as:

$$
K=\frac{1}{n(r . m . s)^{4}} \sum_{j-1}^{n}\left(x_{j}-\bar{x}\right)^{4}
$$

where $x_{j}$ is the amplitude of signal, $n$ is number of data and $\bar{x}$ is the mean value.

\section{The Wavelet Transform}

The wavelet transform (WT) is defined in the time-scale domain and is a significant tool for analysing the time-localised features of a signal. It represents a windowing technique within the variable-sized region. A wavelet transform can be classified as either a continuous wavelet transform (CWT) or a discrete wavelet transform (DWT) depending on the discretisation of the scale parameters of the analysing wavelet. The DWT based on such wavelet functions is called the orthogonal wavelet transform (OWT). Orthogonal wavelet transforms are normally applied for the compression and feature 
selection of signals. DWT is derived from discrete CWT, and it is shown as the following expression, Purushotham et al. [8]:

$$
W_{\psi}(m, n)=\int_{-\infty}^{\infty} x(t) a_{0}^{-m / 2} \psi^{*}\left(a_{0}^{-m}, t-n b_{0}\right) d t
$$

where $a$ and $j$ are the scale factor, both $b$ and $k$ are the position, and $\Psi$ is the mother wavelet. Oh [9] has previously conducted fatigue data analysis using the wavelet transform (WT) for spike removal, denoising, and data editing. Piotrkowski et al. [10] used the Wavelet Transform application in acoustic emissions to detect damage and corrosion.

\section{Fatigue Life Assessment}

The Palmgren-Miner linear cumulative damaging rule is normally associated with the established strain-life fatigue models Sun et al., [11]. The fatigue damage caused by each cycle of repeated loading is calculated by reference to material life curves, such as $S-N$ or $\varepsilon-N$ curves. The fatigue damage caused by multiple cycles is expressed respectively as:

$$
\begin{aligned}
& D=\left(\frac{1}{N_{f}}\right) \\
& \sum D=\sum\left(\frac{N_{i}}{N_{f}}\right)
\end{aligned}
$$

where $D$ is fatigue damage for one cycle and $\sum D$ is total fatigue damage $N_{i}$ is the number of cycles within a particular stress range and its mean and $N_{f}$ is a number of cycles.

The strain-life model commonly used for the prediction of fatigue strain life is the Coffin-Manson relationship model. This model can provide a traditional prediction when there is more compressive load time history and the mean stress is zero. The following equation can define this model:

$$
\varepsilon_{a}=\frac{\sigma_{f}^{\prime}}{E}\left(2 N_{f}\right)^{b}+\varepsilon^{\prime} f\left(2 N_{f}\right)^{c}
$$

$E$ is the material modulus of elasticity, $\varepsilon_{a}$ is the true strain amplitude, $2 N_{f}$ is the number of reversals to failure, $\sigma_{f}^{\prime}$ is the fatigue strength coefficient, $b$ is the fatigue strength exponent, $\varepsilon_{f}^{\prime}$ is the fatigue ductility coefficient, $c$ is the fatigue ductility exponent, $\sigma_{m}$ is the mean stress, and $\sigma_{\max }$ is the maximum stress.

The inclusion of mean stress effects in the life prediction makes the process more complex. The Morrow mean stress model is given by Dowling [12]:

$$
\varepsilon_{a}=\frac{\sigma_{f}^{\prime}}{E}\left(1-\frac{\sigma_{m}}{\sigma_{f}^{\prime}}\right)\left(2 N_{f}\right)^{b}+\varepsilon_{f}^{\prime}\left(2 N_{f}\right)^{c}
$$

where is the total strain amplitude, $\sigma_{f}^{\prime}, \mathrm{b}, \varepsilon^{\prime}{ }_{f}$ and c are considered to be material properties, $N_{f}$ is the number of cycles to failure, and $\sigma_{m}$ is the mean stress. Another strain life model dealing with mean stress effects is known as the SmithWatson-Topper (SWT) model, and its equation is written as:

$$
\varepsilon_{a} \sigma_{m a k}=\frac{\sigma_{f}^{\prime 2}}{E}\left(2 N_{f}\right)^{2 b}+\sigma_{f}^{\prime} \varepsilon_{f}^{\prime}\left(2 N_{f}\right)^{b+c}
$$


The Coffin-Manson relationship only considers the damaging calculation at zero mean stress. However, in real-case scenarios, some of the realistic service situations involve nonzero mean stresses. For example, in a case of the loading being predominantly compressive, particularly for wholly compressive cycles, the Morrow mean stress correction effect provides more realistic life estimates and seems to work reasonably well for steels, Ince \& Glinka [13].

\section{The Weibull Distribution}

In terms of the statistical analysis used in engineering, the Weibull distribution is a theoretical model that has been successfully used to model the life data. The Weibull distribution is described by the shape, scale, and threshold parameters. The Weibull distribution model is a tool to develop the probabilistic analysis because of its ability to provide reasonably accurate failure analysis and failure forecasts with extremely small samples, Sivapragash, [14]. The 2-P Weibull distribution function is shown in Eqn. 7:

$$
f(x: \theta, \beta)=\frac{\beta}{\theta}\left(\frac{x}{\theta}\right) \exp \left[-\left(\frac{x}{\theta}\right)^{\beta}\right]
$$

where $f(x: \theta: \beta)$ represents the probability of strain-life being equal to or less than $x, \theta$ is a scale parameter, and $\beta$ is a shape parameter. $\theta$ and $\beta$ are estimated by observation.

The Weibull distribution has been widely utilised to develop a model of extreme values, such as failure time and fracture strength, Shalabh et al. [15]. The Weibull distribution is a probabilistic analysis which has been used for the determination of static and dynamic mechanical properties of materials. This distribution has the capability to model experimental data with different characters, Li et al. [16].

\section{METHODOLOGY}

I $\mathrm{n}$ this work, the strain signal has been collected from the coil spring in the car suspension system during a road test as shown in Fig. 1 which is collected in macrostrain $(\mu e)$. An established signal, known as SAESUS, is the typical strain history for the suspension system, developed by the Society of Automotive Engineers (SAE), as reported in Oh [9]. Another signal, S1, is a set of experimental strain signals measured at $500 \mathrm{~Hz}$ sampling rate using a strain gauge that is positioned on the coil spring component of a car being driven on a rural road at a velocity of 50 to $60 \mathrm{~km} / \mathrm{h}$. The measurement procedure is reported in their previous work by Yunoh et al. [17]. The fatigue signal was measured at the car coil spring which subjected to the road load service. All data were recorded as strain time histories and Fig. 2 shows the setup of fatigue data measurement during the process. The strain value was measured using strain gauge and it was connected to the specific data logger, for data acquisition purpose. Experimental parameters such as sampling frequency and type of output data were then set using the common data logger interface.

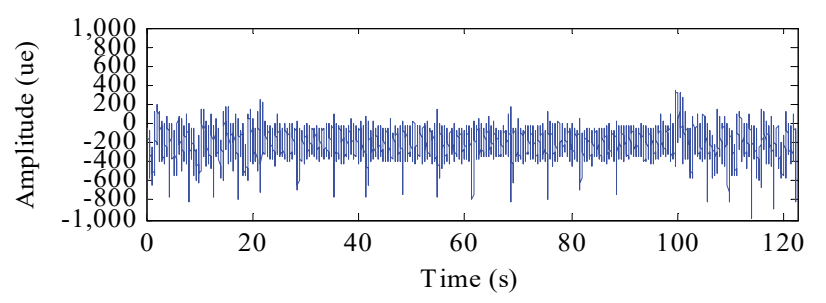

(a)

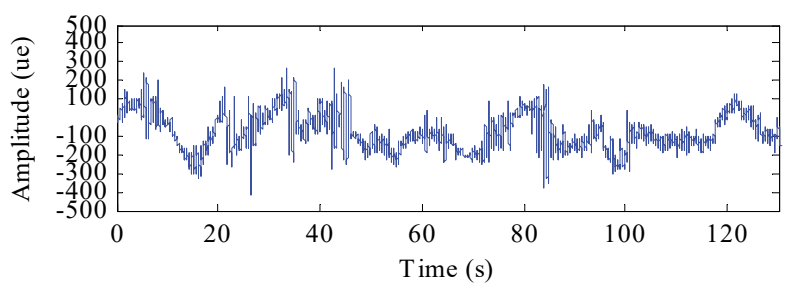

(b)

Figure 1: The strain signals used for the work: (a) SAESUS, (b) An experimental measured data, S1.

The extraction algorithm of the strain signal is developed for fatigue data editing purposes. The discrete wavelet transform is utilised to identify the high amplitude segments in the fatigue signal due to the high-energy coefficients magnitude. The magnitudes of the wavelet energy coefficients in the time-frequency domain are transposed into time histories representations to trace the location of the high amplitude segments. The respective magnitudes are obtained from the accumulation of wavelet transform magnitude distributions along the frequency band at each time interval. Thus, the energy coefficients in time representation are gained, and these signals are used to detect the presence of high amplitude events in the fatigue signal. 
The Cut-off Level (COL) needs to be set up for the elimination process in a high amplitude event extraction process. The $\mathrm{COL}$ is the minimum percentage of the wavelet energy coefficients to be retained and the segments with magnitudes lower than the COL value will be removed. The retained segments are then sliced from the original signal. The sliced segment identification is performed by means of a search which identifies the point at which the signal envelope inverts from the decay behaviour. The two inversion points, one on either side of the peak value, define the temporal extent of the sliced segment. The original strain signal is then edited to remove the low amplitude cycle contained in the signal based on the time location of the wavelet transform spectrum-sliced segment. The fatigue damage for every retained segment is then calculated for the probabilistic analysis.

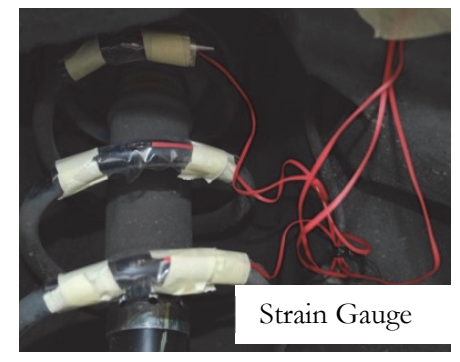

Strain gauge position

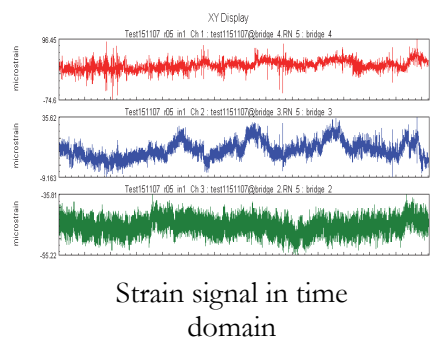

Figure 2: A diagrammatic process flow for fatigue signal collection

The fatigue damage for all segments is further analysed using the 2-P Weibull distribution to evaluate the segments' extraction results. The advantage of using this distribution lies in its ability to explain the simple function. This approach is often used in assessing the fatigue life of the material based on its simple calculation, Glodez et al. [18]. All the fatigue damage of the retained segments is incorporated into the Weibull distribution, and as has been later suggested, based on the probability distribution, the resultant significant findings are utilised to reveal the inferences of this study.

\section{RESULTS AND DISCUSSIONS}

$\mathrm{F}$ irst, the strain signals were evaluated by observation based on statistics characteristics, i.e. number of cycles counted, root-mean-square (r.m.s), kurtosis and total damage as tabulated in Tab. 1. According to the results, the SAESUS signal produced higher values of r.m.s and kurtosis. This is due to the higher amplitude segments existing in the SAESUS signal compared to the S1 signal. Higher amplitude segments contributed more energy in the oscillatory signals. The value of kurtosis for the SAESUS strain signal was found to be 4.32, which indicates that the spike and extreme values exist in the signal. This result was consistent with the r.m.s value for SAESUS, which is found to be $246.6 \mu \varepsilon$, higher when compared to S1. The kurtosis value for both strain signals was not found in a Gaussian distribution because in a stationary Gaussian process, the kurtosis value is approximately 3 .

The fatigue damage for both signals was calculated based on three strain-life models as tabulated in Tab. 1. The values of the fatigue damage based on the three models were close to each other with minor differences. The fatigue damage values for SAESUS were found to be higher compared to S1. The existence of the higher amplitude segments in the SAESUS signal contributed more fatigue damage compared to S1. 


\begin{tabular}{cccclr}
\hline Data & N (Cycles) & r.m.s $(\mu \varepsilon)$ & Kurtosis & & $\begin{array}{r}\text { Fatigue Damage } \\
\text { (Damage/Block) }\end{array}$ \\
SAESUS & 1253 & 246.6 & 4.28 & CF & $1.70 \times 10^{-3}$ \\
& & & & Morrow & $1.70 \times 10^{-3}$ \\
SWT & $1.64 \times 10^{-3}$ \\
S1 & \multirow{2}{*}{122.68} & 2.82 & CF & $2.97 \times 10^{-4}$ \\
& & & & Morrow & $2.94 \times 10^{-4}$ \\
& & & & SWT & $2.85 \times 10^{-4}$ \\
\hline
\end{tabular}

Table 1: Summary of statistical characteristic of the signals

The developed wavelet transform algorithm transformed the signal into a time-frequency domain to obtain the timefrequency localisation, as shown in Fig. 3. The spectrum colour intensity is proportional to the absolute energy coefficients values because it delivers the energy distribution display on time and frequency. The spectrum of SAESUS and S1 signals shows that the intensity colours of the SAESUS strain signal are more highlighted compared to S1. A large scale was indicative of low frequency, and higher amplitude segments indicated that the cycles had higher energy. This means that it can inflict higher fatigue damage.

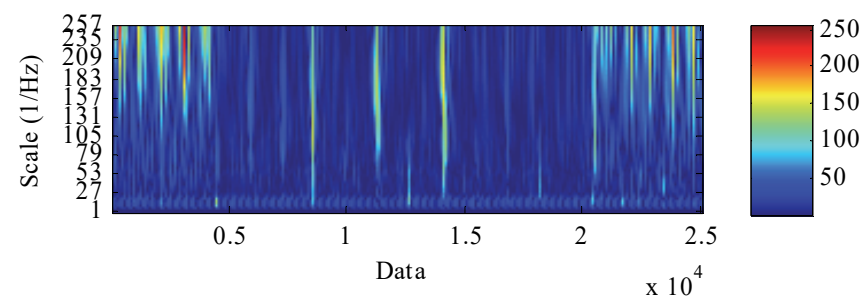

(a)

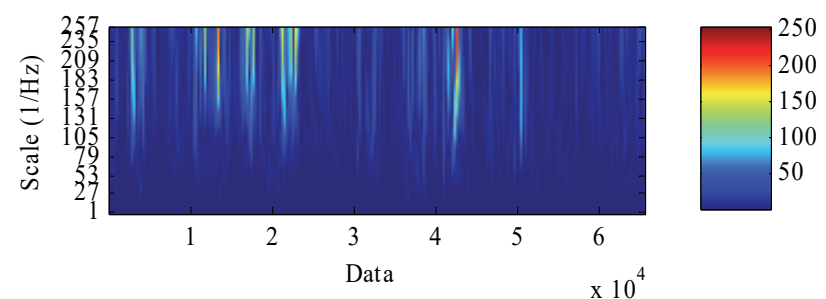

(b)

Figure 3: The wavelet transform spectrum of; (a) SAESUS, (b) S1.

Higher amplitude segments based on wavelet transform spectrum were extracted. The location of the retained segments was shown in Fig. 4. After eliminating the low amplitude segments, the high amplitude segment should be retained to maintain the fatigue damage contained in both signals. The SAESUS strain signal contributed 21 high amplitude retained segments. Meanwhile, the S1 strain signal contributed 11 high amplitude retained segments. Based on the extraction results, the SAESUS strain signal consists of higher amplitude segments compared to S1 because it retained the higher amplitude segments after the extraction process.

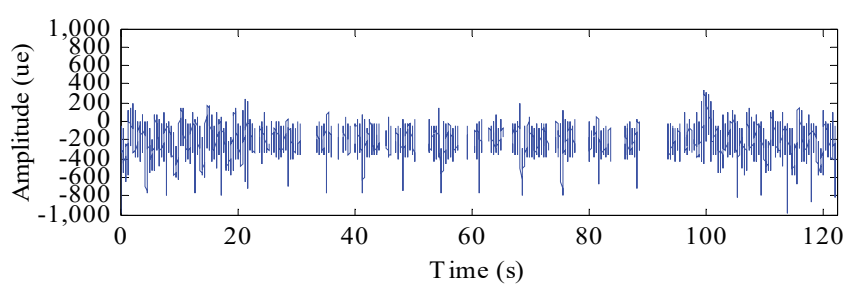

(a)

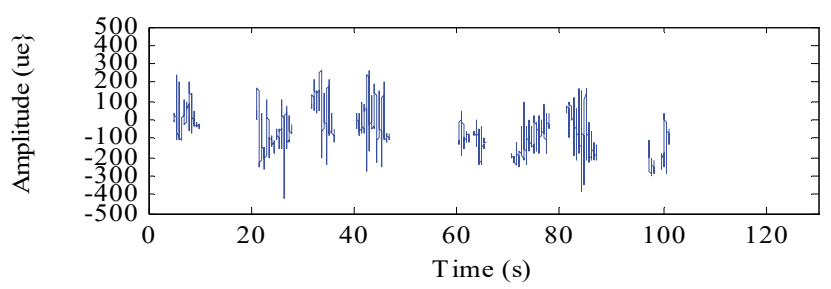

(b)

Figure 4: The location of the retained segments; (a) SAESUS, (b) S1.

The features extraction, i.e. fatigue damage for every segment, was calculated to analyse and determine the probability of failure based on high amplitude segments. It is helpful to know the fatigue damage distribution based on the extracted segments for both signals. The 2-P Weibull distribution was used for the data analysis. According to Tab. 2, the values of the shape parameters, $\beta$, for both signals were less than 1.0, indicating a decreasing failure rate. According to Finkelstein, [19], if the shape parameter for Weibull distribution is less than 1.0, this distribution is always decreasing the failure rate. The value of scale parameter, $\theta$, of fatigue damage for both signals was found to be $5.3 \times 10^{-5}$ and $1.4 \times 10^{-5}$. This means that when the components reach these values, the probability of failure will increase. 


\begin{tabular}{ccc}
\hline Signals & Shape parameter, $\beta$ & Scale parameter, $\theta$ \\
SAESUS & 0.59 & $5.3 \times 10^{-5}$ \\
S1 & 0.34 & $1.4 \times 10^{-5}$ \\
\hline
\end{tabular}

Table 1: Statistical data analysis based on Weibull distribution.

Cumulative Distribution Function (CDF) represents the probability of the components to fail at the particular time interval. Based on the plots in Fig. 5, the fatigue damage for S1 increases more rapidly in cumulative probability values compared to the SAESUS. This finding can be suggested that SAESUS signal consists high amplitude segments that cause high fatigue damage compared to S1.

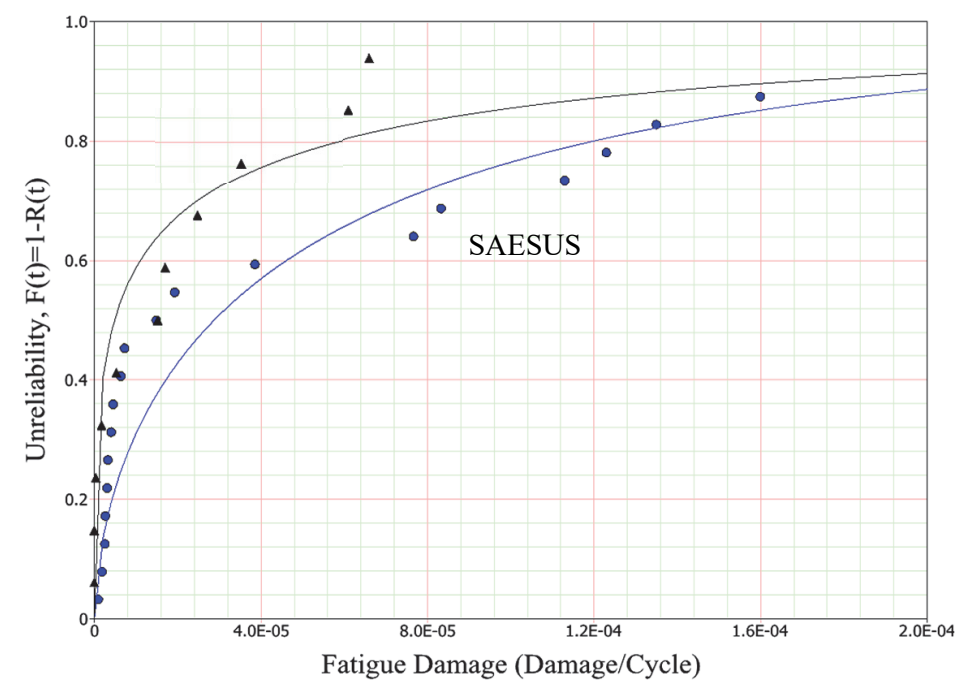

Figure 5: Probability density functions for SAESUS and S1 strain signal

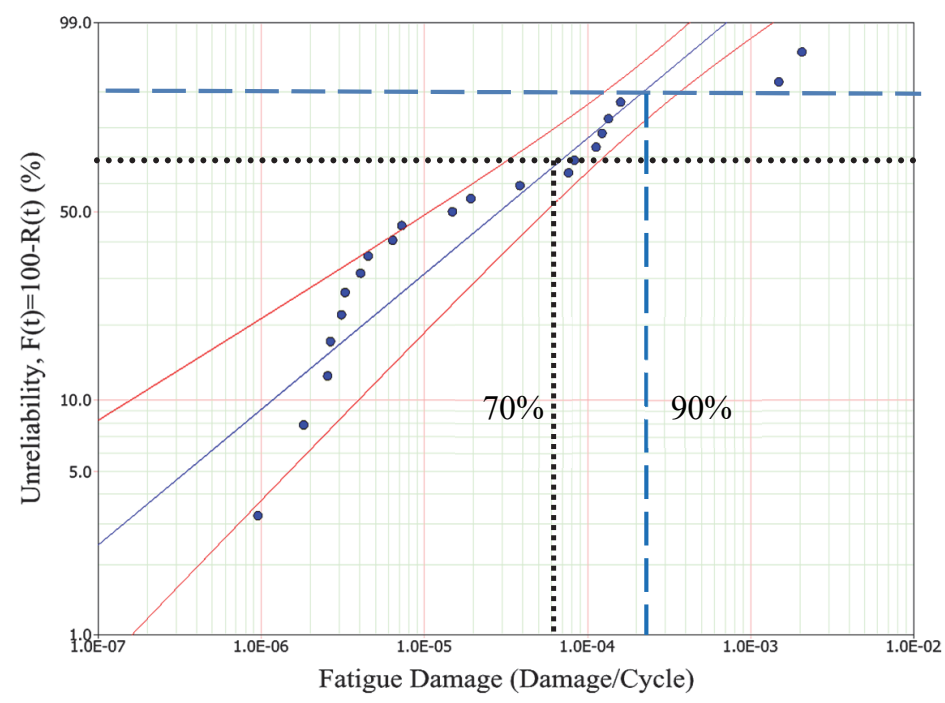

Figure 6: The Weibull probability distribution plots of fatigue damage for SAESUS

Figs. 6 and 7 show the best fit of the Weibull probability distribution plot for fatigue damage of high amplitude segments for both signals. The parameters of the distribution are estimated using the maximum likehood method. The Weibull probability distribution plot for both strain signals was estimated using $95 \%$ confidence level correction line, which includes the true population parameter. From a statistical point of view, the more test data that falls into the $95 \%$ confident level, the more reasonable the probabilistic distribution is, according to Xianmin et al [20]. 
Based on the plot indicated in Fig. 5 for the SAESUS strain signal, there are four data points which are out of the 95\% confidence level. This is because several segments in the signal consist of very high amplitude segments. Meanwhile, all points in S1 strain signal were distributed at the 95\% confidence level. Figs. 5 and 6 also show that 70\% of the probability of failure occurred from $1.0 \times 10^{-5}$ to $1.0 \times 10^{-4}$ fatigue damage for both signals. Around $90 \%$ of the probability of failure occurred from $1.0 \times 10^{-4}$ to $1.0 \times 10^{-3}$ fatigue damage for both signals.

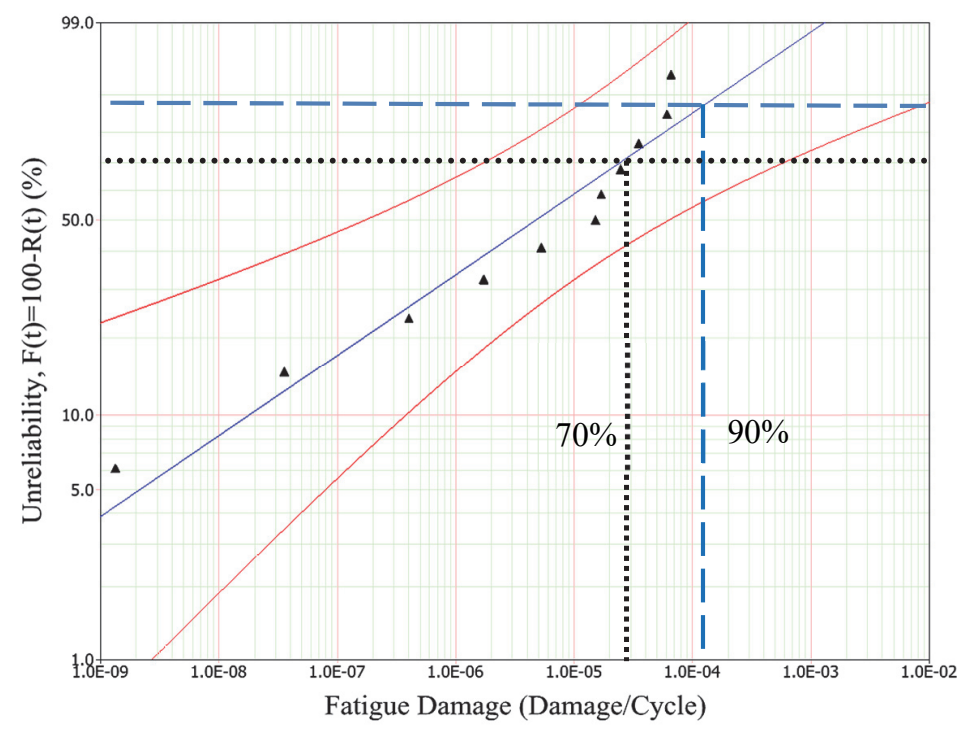

Figure 7: The Weibull probability distribution plots of fatigue damage for S1.

Figs. 8 and 9 show the best fit of the Weibull probability distribution plot for fatigue life of high amplitude segments for both signals. All of data for the SAESUS and S1 strain signal were distributed at the 95\% confidence level. Fig. 7 show that $70 \%$ of the probability of failure for SAESUS occurred at $5.2 \times 10^{3}$ cycles. Meanwhile the probability of failure for SAESUS occurred at $6.9 \times 10^{3}$ cycles. Around $90 \%$ of the probability of failure occurred at $1.6 \times 10^{5}$ to $3.5 \times 10^{5}$ fatigue life for both signals. Thus, the value of fatigue damage and life based on extraction segments approaching the failure can be determined roughly by the Weibull analysis.

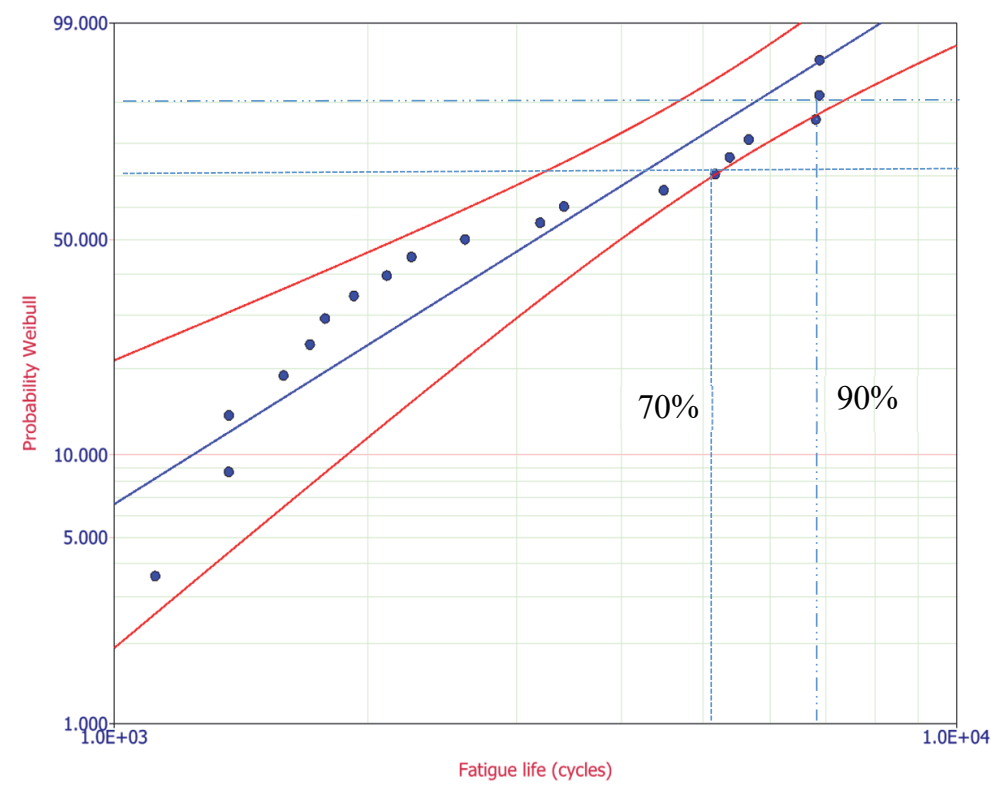

Figure 8: The Weibull probability distribution plots of fatigue damage for SAESUS. 


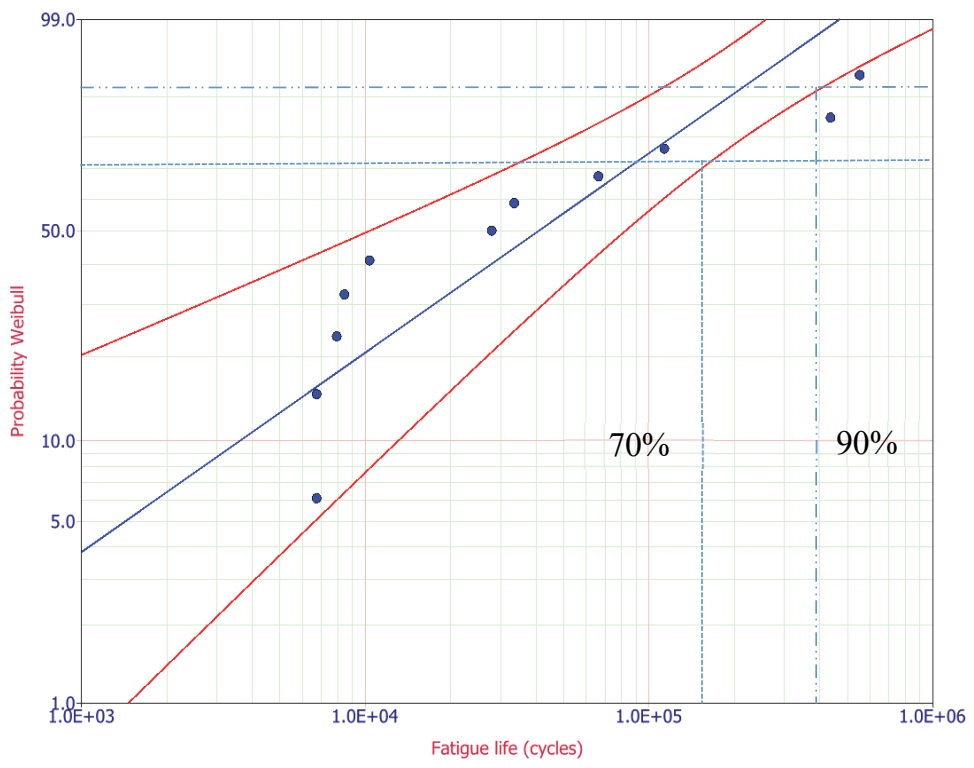

Figure 9: The Weibull probability distribution plots of fatigue damage for S1.

\section{CONCLUSION}

$\mathrm{T}$ o summarise the statistics and strain life analysis, the SAESUS strain signal contributes more damaging segments compared to S1. This situation is due to the high amplitude segments obtained in the signal. The fatigue damage based on high amplitude segments fits well to a Weibull distribution. Based on the results, it shows that $70 \%$ of the probability of failure occurred between $1.0 \times 10^{-5}$ and $1.0 \times 10^{-4}$ fatigue damage for both signals. Around $90 \%$ of the probability of failure occurred between $1.0 \times 10^{-4}$ to $1.0 \times 10^{-3}$ fatigue damage for both signals. Thus, the value of fatigue damage based on extraction segments that are approaching the failure can be determined using the Weibull analysis with the $95 \%$ confident level, which is a reasonable probabilistic distribution. This approach presents an alternative technique to predict the fatigue damage probability function for automotive components. However, further works and analyses need to be performed for the purpose of validation, and also to produce high data accuracy by means of life assessment.

\section{REFERENCES}

[1] Sonsino, C. M. (2006). Fatigue testing under variable amplitude loading, International Journal of Fatigue, 29(6), pp. 1080-1089.

[2] Stephens, R. I., Dindinger, P. M., and Gunger, J. E. (1997). Fatigue damage editing for accelerated durability testing using strain range and SWT parameter criteria, International Journal of Fatigue, 19(8-9), pp. 599-606.

[3] Abdullah, S., Nizwan, C. K. E., Yunoh, M. F. M., Nuawi, M. Z., and Nopiah, Z. M. N. (2013). Fatigue features extraction of road load time data using the S-Transform, International Journal of Automotive Technology, 14(5), pp. 805-815.

[4] El-Ratal, W., Bennebach, M., Lin, X. and Plaskitt, R. Fatigue life modelling and accelerated test for components under variable amplitude loads, Proc. of Symposium on Fatigue Testing and Analysis under Variable Amplitude Loading Conditions, Tours, France, 2002.

[5] Nizwan, C. K. E., Abdullah, S., Nuawi M. Z. and Lamin, F. (2007). A study of fatigue data editing using frequency spectrum filtering technique, Proceeding of the World Engineering Congress (WEC), Penang, Malaysia, 2007, pp. 372378.

[6] Zhao, Y. X. and Liu, H. B. (2014). Weibull modelling of the probabilistic S-N curves for rolling contact, International Journal of Fatigue, 66, pp. 47-54.

[7] Tiryakioglu, M. (2015). Weibull analysis of mechanical data for casting II: Weibull mixtures and their interpretation, Metallurgical and Materials Transactions A, 46A, pp. 270-280. 
[8] Purushotham, V., Narayanan, S. and Prasad, S. A. N. (2005). Multi-fault diagnosis of rolling bearing elements using Wavelet analysis and hidden Markov model-based fault recognition, NDT \& E International, 38, pp. 654-664.

[9] Oh, C. S. (2001). Application of Wavelet transform in fatigue history editing, International Journal of Fatigue, 23(3), pp. 241-250.

[10] Piotrkowski, R.,Castro, E and Gallego, A. (2009). Wavelet power, entropy and bispectrum applied to AE signals for damage identification and evaluation of corroded galvanised steel, Mechanical System and Signal Processing, 23(2), pp. 432-445.

[11] Sun, Q., Dui, H. N. and Fan, X. L. (2014). A statistically consistent fatigue damage model based on Miner's rule, International Journal of Fatigue, 69, pp. 16-21.

[12] Dowling, N. E. (2013). Mechanical Behavior of Materials, 4th ed.: Pearson Education Limited.

[13] Ince, A. and Glinka, G. A modification of Morrow and Smith-Watson-Topper mean stress correction models, Fatigue Fracture Engineering Materials and Structure, 34, pp. 854-867.

[14] Sivapragash, M., Lakshminarayanan, P. R., Karthikeyen, R., Raghukanda, K. and Hanumantha, M. (2008). Fatigue life prediction of ZE41A magnesium alloy using Weibull distribution, Material \& Design, 29 pp.1549-53.

[15] Shalabh G, Dheeraj S.S, Asok R. Statistical pattern analysis of ultrasonic signals for fatigue damage detection in mechanical structures, NDT \& E International, 41, pp. 491-500.

[16] Li W, Sakai T, Wang P. (2011). Statistical analysis of fatigue crack growth behavior for grade B cast steel. Material and Design, 32, pp. 1262-1272.

[17] Yunoh, M. F. M., Abdullah, S., Saad, M. H. M., Nopiah, Z. M. and Nuawi, M. Z. (2014). Fatigue time history analysis for determining the strain signal behavior, International Journal Vehicle System Modelling and Testing, 9 (3/4), pp.363371.

[18] Glodez, S., Sori, M., and Kramberger. J. (2013). Prediction of micro-crack initiation in high strength steels using Weibull distribution. Engineering Fracture Mechanics, 108, pp. 263-274.

[19] Finkelstein, M. (2009). Understanding the shape of the mixture failure rate (with engineering and demographic applications), Applied Stochastic Models in Business and Industry, 25(6): 643-663.

[20] Xianmin, C., Qin, S., Hongna, D. and Junling, F. (2016). A statistically self-cnsistent fatigue damage accumulation model including load sequence effects under spectrum loading, Frattura ed Integrita Strutturale, 38, pp. 319-330. 